\title{
Study on Hybrid PWM Method under Low Switching Frequency
}

\author{
Wei Kekang*, Trillion Q . Zheng*, Ran Wang*, and Chenchen Wang*
}

\begin{abstract}
This paper presents a hybrid pulse width modulation (PWM) method under low switching frequency conditions based on space vector PWM (SVPWM) and selective harmonic eliminated PWM (SHEPWM), which use asynchronous carrier modulation SVPWM at low frequency, and SHEPWM at high frequency, a square wave after rated conditions. A transitive strategy is proposed to realize a smooth transition of individual modes including SVPWM, SHEPWM and square waves. Experimental results confirm this hybrid modulation method and their transition are reasonable and proper.
\end{abstract}

\section{Keywords: Hybrid PWM, Low Switching Frequency, SVPWM, SHEPWM}

\section{Introduction}

Differing from general industrial drive systems, railway traction drive systems, applied to AC-DC-AC electric locomotives, Electric Multiple Units (EMU), the subway car and so on, have the following characteristics: on the one hand the maximum switching frequency is generally a few hundred Hertz; on the other hand, when the output reaches the rated conditions, it works in the square wave. Therefore, in the entire speed range, the carrier ratio range is very large. As such, the designers of PWM strategies need to consider the following factors:

1) The range of each pulse width modulation method to make the inverter output voltage continuous and reach the square wave (one pulse) condition;

2) The current harmonics content, current peak and loss of the system is acceptable.

Generally speaking, the pulse width modulation strategy of railway traction converters is multi-mode PWM; that is, asynchronous SVPWM (or SPWM) is adopted at the low frequency phase, synchronous SPWM is mainly adopted at the intermediate frequency stage, and one pulse is used at the high frequency stage full use of DC-link voltage. The three phase Space Vector PWM (SVPWM) has some merits, such as easy-to-digitalize implementation, low torque ripple, low noise and high voltage utilization. This is because the inverter and motor are regarded as a whole unit.

\footnotetext{
School of Electrical Engineering, Beijing Jiaotong University, China (07117332@bjtu.edu.cn, tqzheng@ieee.org)

Received 29 June 2011; Accepted 14 November 2011
}

Therefore, SVPWM is widely used in general industrial drives. At the low frequency stage, as the carrier ratio of PWM is high, the subharmonics introduced by the positive and negative half-cycle asymmetry of asynchronous modulation can be ignored. The asynchronous modulation does not transition between the different carrier ratios of synchronous PWM, and makes full use of the inverter switch capacity. However, the influence of subharmonics is greater as the carrier ratios become lower at the asynchronous PWM stage, which should transit to a synchronous SPWM strategy. When the carrier ratio becomes lower than 11, the low index harmonics are greater and it becomes hard to transition to one pulse modulation, especially when the carrier ratios equal three. Therefore the PWM strategy should adopt a special method to get better harmonic features and achieve symmetry with the current waveform. In this way, the inverter can get in square-wave mode without dash current.

Special modulation is also a synchronous modulation whose waveform possesses three-phase symmetry, half-wave symmetry and quarter-wave symmetry to keep the harmonics and their undesirable effects to a minimum. It is also a synchronous modulation. In this case the switching angles are optimized offline. These switching angles' objected function is a certain output characteristic, such as total harmonic distortion (THD) of current, or torque ripple minimization. Selective Harmonic Eliminated PWM (SHEPWM), one of the earliest forms of PWM, aims to progressively eliminate low-order harmonics. However, its disadvantage is that it's hard to calculate in real-time and needs a large 
storage space when its carrier ratios are high.

In this paper, a hybrid PWM method based on SVPWM and SHEPWM suited for a railway high power low switch frequency converter is presented. First, the principle and realization of SHEPWM is introduced. Second, the transitive strategy between different modulations is analyzed. Finally, the method is verified by simulation and experiment.

\section{The Principle and Realization of Hybrid PWM}

The hybrid PWM method can be broken into three parts: SVPWM, SHEPWM and square wave mode. Square wave is very simple and easy to carry out, and is described in many papers. As such, it is not discussed further in this paper. Though it is one of the earliest forms of PWM, SHEPWM is a special PWM mode which is described in detail here.

\subsection{The Principle of SVPWM}

The principle of SVPWM is based on the fact that there are only 8 possible switch combinations (including six active vectors and two zero vectors) for a three-phase inverter as shown in Fig.1. An arbitrary target output voltage vector $U_{\text {out }}$ can be formed by the summation (averaging) of the two nearest vectors and zeros vectors within one switching period.

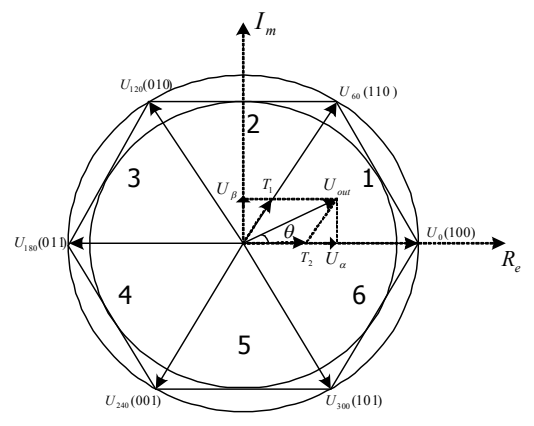

Fig. 1. Basic voltage vectors of a two-level inverter

As an example, the voltage vector $U_{\text {out }}$ in the first sector is composed of two active vectors $(100,110)$ and zero vectors as shown in Fig.1. The duty of each active vector can be expressed mathematically as

$$
\left\{\begin{array}{c}
T_{1}=\sqrt{3} \frac{U_{m}}{U_{d c}} \cdot T_{P W M} \cdot \sin \left(\frac{\pi}{3}-\theta\right) \\
T_{2}=\sqrt{3} \frac{U_{m}}{U_{d c}} \cdot T_{P W M} \cdot \sin \theta
\end{array}\right.
$$

Here, $U_{m}$ is the amplitude of voltage vector $U_{\text {out }}, U_{d c}$ is a DC-bus voltage, $\theta$ is the angle of voltage vector $U_{\text {out }}, T_{P W M}$ is one switching period.

It follows that the duty of zero vectors can be expressed as

$$
T_{0}=T_{P W M}-T_{1}-T_{2}
$$

\subsection{The Principle and Realization of SHEPWM}

The basic ideal of SHEPWM is that the $\mathrm{N}$ chops the switching distribution of the inverter's waveform as determined by solving the nonlinear equations simultaneously, based on Fourier analysis. The waveforms in Fig. 2 can be represented in general by a Fourier series as

$$
f(\omega t)=\sum_{n=1}^{\infty}\left[a_{n} \sin (n \omega t)+b_{n} \cos (n \omega t)\right]
$$

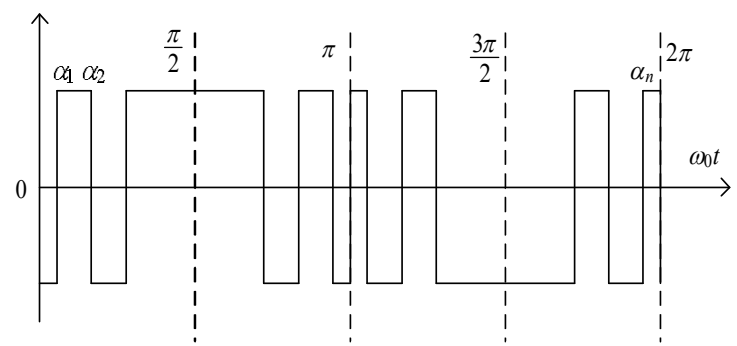

Fig. 2. Generalized output waveform of the inverter

Because of its half-wave symmetry and quarter-wave symmetry, Equal. (4) can be obtained as follows

$$
\left\{\begin{array}{l}
f(\omega t)=\sum_{n=1,3,5, \cdots}^{\infty} a_{n} \sin (n \omega t) \\
a_{n}=\frac{2 U_{d}}{n \pi}\left[1+2 \sum_{j=1}^{M}(-1)^{j} \cos \left(n \alpha_{j}\right)\right]
\end{array}\right.
$$

Here $\alpha_{1}, \alpha_{2} \ldots \alpha_{M}$, define the angular position of the waveform as shown in Fig.2.

When SHEPWM is applied to a three-phase traction motor driver system, there only exists non-triplen odd harmonics, and triplen odd harmonics are not considered in the harmonics eliminated process. Therefore, when there are $\mathrm{M}$ notches in the quarterwave, one function is selected to determine the fundamental voltage value. Namely other functions are selected to eliminate $6 k \pm 1$ harmonics, that is

$$
\left\{\begin{array}{l}
\left|U_{m(1)}\right|=\mid \frac{4}{\pi}\left[1+2 \sum_{j=1}^{M}(-1)^{j} \cos \left(\alpha_{j}\right)\right]=m_{d} \\
U_{m(n)}=\frac{4}{n \pi}\left[1+2 \sum_{j=1}^{M}(-1)^{j} \cos \left(n \alpha_{j}\right)\right]=0
\end{array}\right.
$$

Here $m_{d}=2 u_{1} / u_{d c}$, and $\alpha_{1}<\alpha_{2}<\alpha_{j}<\cdots<\alpha_{M}<\pi / 2$ 
The set of equations given by Eq.(5) are nonlinear since they are trigonometric function of the variable $j$, which is hard to solve. Internationally, many experts proposed solutions, such as the Newton-Raphson method, and the homotopy algorithm. There are no essential differences between these methods, which are difficult to achieve through real-time computation. In this paper, the approach to solving these equations is proposed by a Matlab nonlinear function (fsolve())

After eq. (5) is solved by Matlab for several depths of modulation, a simple function about the relationship between modulation depths and switch angles is established by a polynomial fitting method, as showed in eq.(6). The polynomial result of the whole modulation index is shown in Fig.3.

$$
\alpha_{k}=a x^{3}+b x^{2}+c x+d
$$

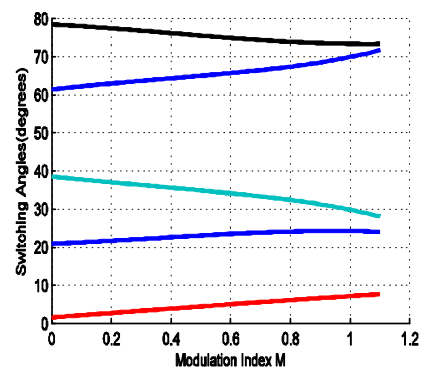

(a) angle vs. modulation index

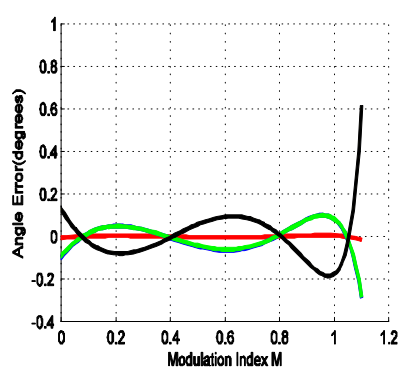

(b) angle errors vs. modulation index
Fig. 3. Polynomial Result

The realization of a carrier-based PWM by a digital signal processor (DSP) or another more commonly used digital controller, is to link a timer to a set of comparison registers that trigger an event when the timer count matches the comparison register contents. Each comparison register controls one phase leg, usually with some associated output logic so that the comparison event can force a phase leg status of high, low, toggled or off. The sawtooth, or triangular carrier modulation can be easily implemented with this system, either by loading the comparison registers with new values every half carrier interval as before, or more easily, by arranging the timer to count 'up' and then 'down' over successive half carrier intervals, and toggling the logical output from the comparison register as the timer count passes the compare register value 'upwards' and 'downwards', as shown in Fig.4. Nevertheless, SHEPWM is not a kind of carrier-based PWM form, which cannot be directly generated by a DSP's timer in common with a carrier-based PWM, such as SVPWM or SPWM. The waveform of SHEPWM is realized by the DSP's PWM generator module, whose parameters are updated over a constant period, reducing the PWM maintenance time and software consumption, as shown in Fig.5.

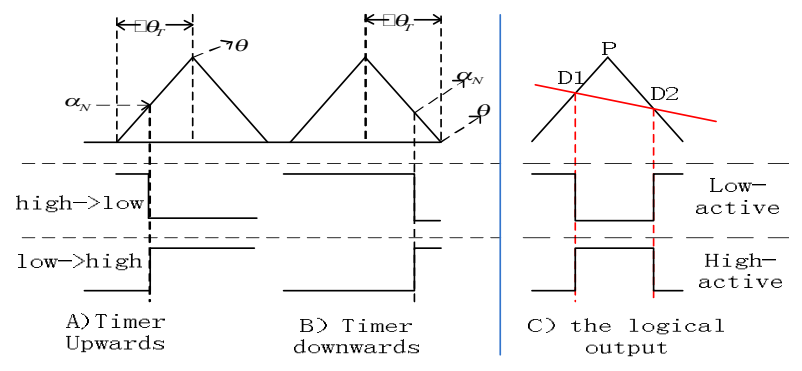

Fig. 4. Diagram of digital PWM generation

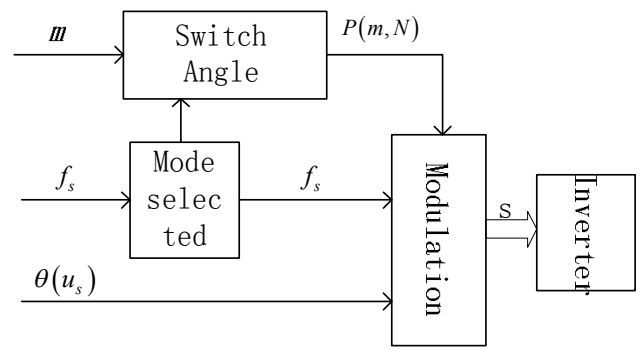

Fig. 5. Realize diagram of SHEPWM

In Fig.5, the SHEPWM mode is first selected by the stator frequency $f_{s}$. After this, the selected SHEPWM mode and the modulation index $\mathrm{m}$, which is proportional to the fundamental voltage magnitude $\left|u_{s}\right|$, are determined by the switching angles series $P(m, N)$. Finally, the phase of the fundamental voltage, stator frequency and switching angles series are fed into a modulation module. The modulation module converts switching angles $\alpha_{j}$ to discrete switching state vectors, which require the phase angle $\theta\left(u_{s}\right)$ of the reference voltage vector as an input to appropriately place the switching transitions within the fundamental period. The fundamental frequency translates the resulting switching angles $\alpha_{j}$ to switching instants described in eq. 7 .

$$
\left\{\begin{array}{cc}
D=\mathrm{P}\left(1-\left(\theta-\alpha_{N}\right) / \Delta \theta_{T}\right) & \cdots \text { Upwards } \\
D=\mathrm{P}\left(\left(\theta-\alpha_{N}\right) / \Delta \theta_{T}\right) & \cdots \text { Downwards }
\end{array}\right.
$$

Here, $\mathrm{P}$ is the period value of the timer, $\theta$ is a step value of the fundamental frequency at timer interval Ts. $\alpha_{N}$ is the switching angle value $\Delta \theta_{T}=2 \pi f_{s} T_{s}$.

\section{The Transition between Different Modulations}

One of the key problems of hybrid PWM is that when switching between SVPWM and SHEPWM at improper angles, there a big dash current or even 
oscillation should be produced. This paper analyzes the motor model and finds the key factors which produce this impulse current.

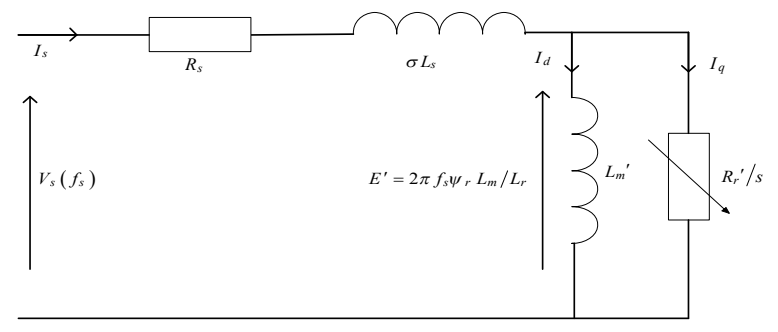

Fig. 6. Equivalent circuit of induction motor

As shown in Fig.6, the relationship between the voltage and current of the induction motor fed by an inverter is as follows:

$$
V_{s}=R_{S} \cdot \mathbf{i}_{s}+\sigma L_{s} \frac{d \mathbf{i}_{s}}{d t}+\mathbf{E}^{\prime}
$$

Here, $\boldsymbol{V}_{s}$ is the voltage vector, $\boldsymbol{i}_{\mathrm{s}}$ is the current vector, $\boldsymbol{E}^{\prime}$ is the voltage affected by the rotor flux which is a slow variation supposed to have only a fundamental vector. Both $\boldsymbol{V}_{\mathrm{s}}$ and $\boldsymbol{i}_{\mathrm{s}}$ are supposed to have a fundamental wave and harmonics.

According to the linear superposition principle, the total response of the equivalent circuit can be expressed as eq.(9)

$$
i_{s}(t)=\sum_{n=1}^{\infty} i_{n}^{\prime}(t)+\sum_{n=1}^{\infty}\left(i_{n}\left(0_{-}\right)-i_{n}^{\prime}\left(0_{+}\right)\right) e^{-t / \tau_{n}}
$$

Here $i_{n}^{\prime}(t)$ is the steady-state current after the PWM transition. $i_{n}\left(0_{-}\right)$is the current value under the old PWM mode and $i_{n}^{\prime}\left(0_{+}\right)$is the current value under the new PWM mode at the transitive point. As can be seen from eq.(9), when the currents of the previous and new PWM modes at the transitive point are not similar, a current strike will occur. The steady-state current can be expressed as follows:

The current value is proportional to the fundamental voltage magnitude and inversely proportional to the harmonic index.

$$
\begin{aligned}
i_{n}{ }^{\prime}(t) & =\sum_{n=1}^{\infty} \frac{\left|V_{s n}\right|}{\sqrt{R_{s}{ }^{2}+\left(n \omega_{s} \sigma L_{s}\right)^{2}}} \cos \left(n \omega_{s} t+\theta_{n}\right) \\
& -\frac{\left|E^{\prime}\right|}{\sqrt{R_{s}{ }^{2}+\left(\omega_{s} \sigma L_{s}\right)^{2}}} \cos \left(\omega_{s} t+\phi\right)
\end{aligned}
$$

The fundamental voltage is a controlled variable which ensures the fundamental current to be continuous, but the harmonics are uncontrolled and parasitic at the normal PWM mode. Therefore, the transitive point is chosen at the moment that the main harmonic current is zero and there is a continuous phase of fundamental current.

According to eq.(10), the harmonic current between the two PWM modes will be similar when the voltage phase is at $\pi / 2$ and $3 \pi / 2$. Therefore a different SHEPWM mode should be selected at these points. When the transition between SVPWM and SHEPWM happens, the harmonics are not considered, for the lowest harmonic index is high and the lowest harmonics of SHEPWM (ratio of carrier/fundament is 11) is 17 .

The transition between SHEPWM and square wave modulation is very easy, as shown in Fig.8. As the fundamental voltage magnitude increases, the switching angle of SHEPWM decreases. When the switching angle reaches zero, the square wave modulation occurs.

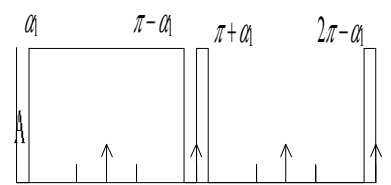

(a)waveform

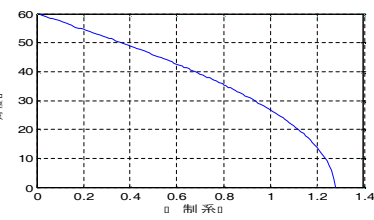

(b) angle vs modulation index
Fig. 7. $\mathrm{SHEPWM}(\mathrm{CR}=3)$

In addition, hysterics are added at the transitive point to void slight oscillation. The hysteresis width is $0.5 \mathrm{~Hz}$.

\section{Experiment Results}

The experiment is designed to verify the key point of SHEPWM in the analysis above. The parameters of the induction motor in simulation and experimentation are listed in Tab.1. The experimental controller is a dual DSP controller board, based on TMS320VC33 and TMS320LF2407.

Table 1. Parameter of induction motor

\begin{tabular}{cccc}
\hline Parameter & Value & Parameter & Value \\
$P / \mathrm{kW}$ & 300 & $V_{s} / \mathrm{V}$ & 2000 \\
$F_{s} / \mathrm{Hz}$ & 68 & $I_{s} / \mathrm{A}$ & 108 \\
$L_{s l} / \mathrm{mH}$ & 2.0317 & $L_{r l} / \mathrm{mH}$ & 2.7921 \\
$R_{r} / \Omega$ & 0.24465 & $R_{s} / \Omega$ & 0.18591 \\
$L_{m} / \mathrm{mH}$ & 60.6639 & & \\
\hline
\end{tabular}

The dc-link voltage is $630 \mathrm{~V}$. Because the DC-link voltage is limited, the experiment is designed based on reduced power. The whole PWM scheme is presented in Fig.8: the asynchronous SVPWM is selected below 
$36 \mathrm{~Hz}$, the SHEPWM $(\mathrm{CR}=11)$ is selected between $36 \mathrm{~Hz}$ and $46 \mathrm{~Hz}$, the SHEPWM $(\mathrm{CR}=7)$ is selected between $46 \mathrm{~Hz}$ and $56 \mathrm{~Hz}$, the SHEPWM $(\mathrm{CR}=5)$ is selected between $56 \mathrm{~Hz}$ and $65 \mathrm{~Hz}$, he SHEPWM $(\mathrm{CR}=3)$ is selected between $65 \mathrm{~Hz}$ and $68 \mathrm{~Hz}$, and the square wave modulation is selected high than $68 \mathrm{~Hz}$.

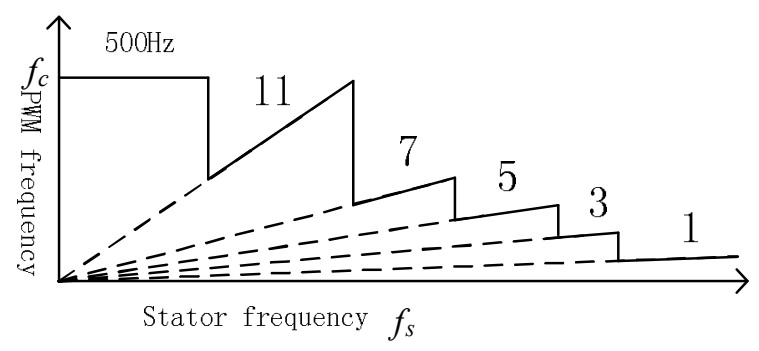

Fig. 8. The Proposed PWM Method Map

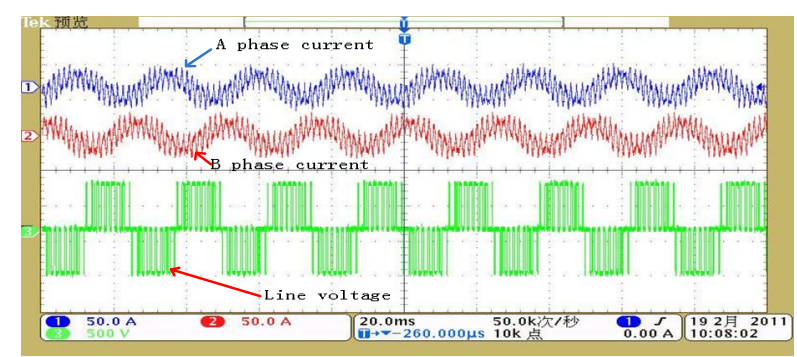

(a) Voltage waveform

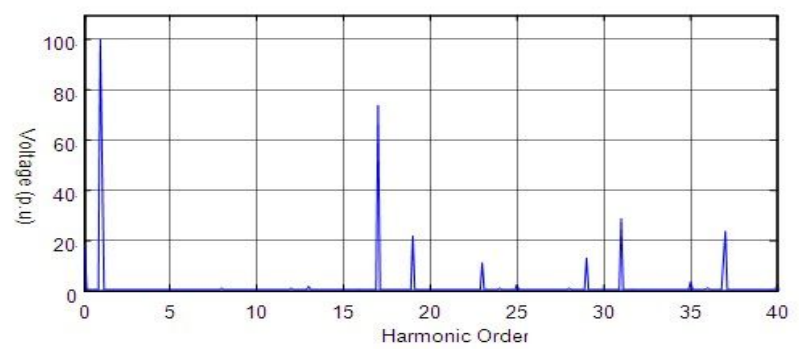

(b) Frequency spectrum

Fig. 9. Waveform and frequency spectrum of SHEPWM $(\mathrm{CR}=11)$

As the space of this paper is limited, some experimental results, and a motor acceleration condition are shown here. The voltage waveform and frequency spectrum of SHEPWM $(\mathrm{CR}=11)$ are shown in Fig.9. As seen from Fig.9., the low harmonics order voltages have been eliminated.

The experimental results of the transition between different PWM methods are shown in Fig.10. In each picture, from the top to bottom in order, there are two phase currents (phase A and B) and line voltages. The dotted lines outline the transition duration between different PWM methods. Seen from each picture, the current impact is very small.

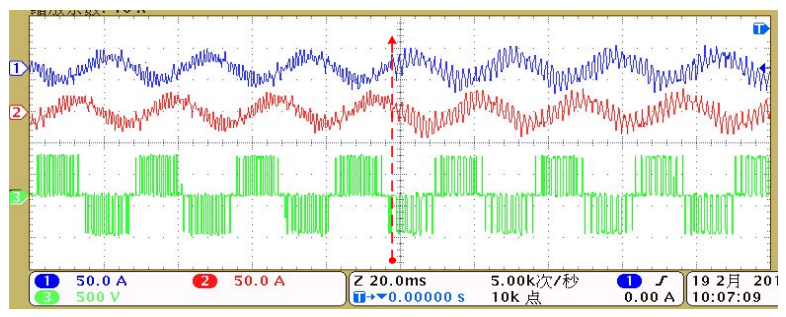

(a) SVPWM jump to SHEPWM $(\mathrm{CR}=11)$

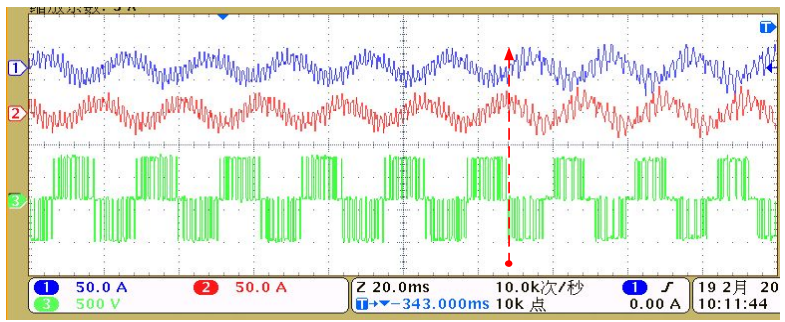

(b) SHEPWM (CR=11)jump to SHEPWM $(\mathrm{CR}=7)$

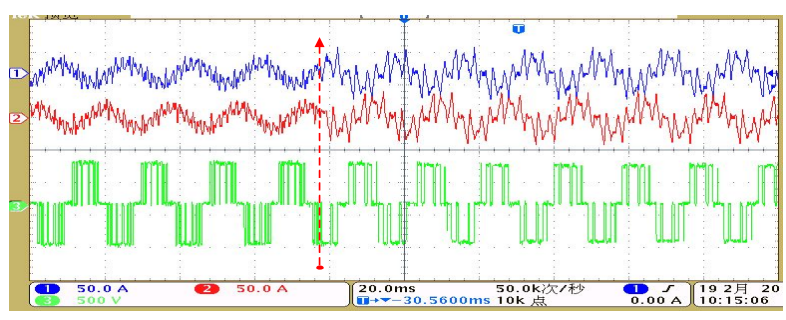

(c) SHEPWM (CR=7)jump to SHEPWM $(\mathrm{CR}=5)$

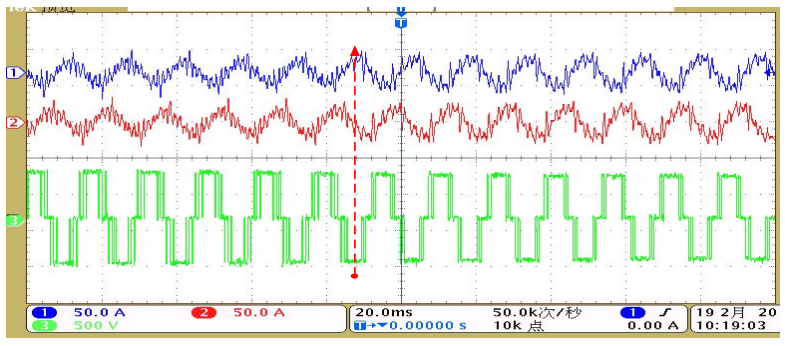

(d) SHEPWM (CR=5)jump to SHEPWM ( $\mathrm{CR}=3)$

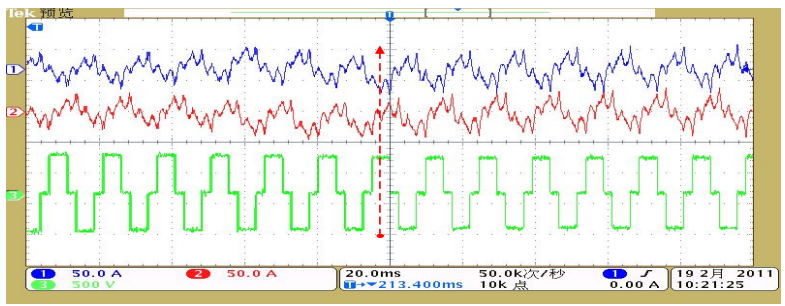

(e) SHEPWM $(\mathrm{CR}=3)$ jump to square wave

Fig. 10. Experimental results of transition between different PWM methods

\section{Conclusions}

This paper proposed a hybrid PWM scheme based on SVPWM and SHEPWM for a railway traction converter operating with low switching frequency. The 
digital realization of SHEPWM and the transitive strategy between different PWM methods are discussed. Experimental results confirm that this hybrid modulation method and its transition are reasonable and correct.

\section{References}

[1] D. Grahame Holmes, Thomas A. Lipo, "Pulse width modulation for power converters: principles and practice," USA Wiley-IEEE Press 2003.

[2] Wells, J.R., Nee, B.M., Chapman, P.L., Krein, P.T., "Selective harmonic control: a general problem formulation and selected solutions" Power Electronics, IEEE Transactions on. vol.20,no.6, pp.1337-1345,2005.

[3] Holtz, J., "Pulsewidth modulation for electronic power conversion," Proceedings of the IEEE, vol.82,no. 8 ,pp. 1194-1214,1994.

[4] Vladimir Blasko, "Analysis of a hybrid PWM based on modified space-vector and triangle-comparison methods," IEEE Transactions on Industry Applications, vol.33, no.3,pp.756-764, ,1997.

[5] S.R. Bowes, "Regular sampled harmonic elimination PWM control of inverter drives," IEEE Transaction On Power Electronics. vol 10, no.5 pp.521-531,1995.

[6] S.R. Bowes, D. Holliday, "Optimal regular-sampled PWM inverter control techniques," IEEE Transactions on Industrial Electronics, vol.53,no.3,pp. 1547$1559,2007$.

[7] M.S.A. Dahidah, V.G. Agelidis, "Selective harmonic elimination PWM control for cascaded multilevel voltage source converters: a generalized formula[J]," IEEE Transactions on Power Electronics, vol.23 no.4,pp.1620-1630, 2008.

[8] J.R. Wells, B.M. Nee, P.L. Chapman, P.T. Krein, "Selective harmonic control: a general problem formulation and selected solutions[J]," IEEE Transactions on Power Electronics, vol.20 no.6,pp.1337-1345, 2005.

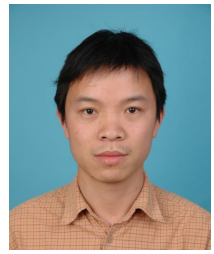

Wei Kekang received his B.S degree in electrical engineering from Beijing Jiaotong University. His research interest is railway electric machines.

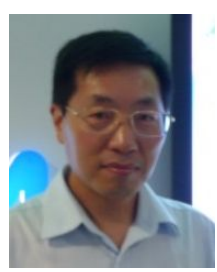

Trillion Q. Zheng is a professor and the dean of School of Electrical Engineering, Beijing Jiaotong University (BJTU). Since 2003, he has led a research team of Power Electronics and Electric Traction, and $\mathrm{He}$ has received numerous achievement awards, including Youth Award of Railway Science \& Technology of Zhan Tianyou (National Level) in 2005, Science \& Technology Award of the Ministry of Railway of China in 1999 and Elite Talent of Youth Science \& Technology of the Ministry of Railway of China in 1998.

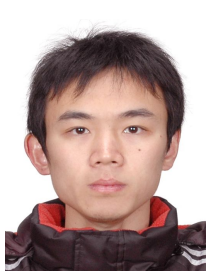

Ran Wang received his B.S degree in electrical engineering from Beijing Jiaotong University. His research interests is railway electric machines.

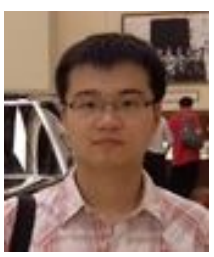

Chenchen Wang is a lector of School of Electrical Engineering, Beijing Jiaotong University (BJTU). $\mathrm{He}$ received his BS, and Ph.D from Tsinghua University. His research interests are high power converter control and indunction motor drive. 